\title{
Perfil da produção de flores tropicais no estado do Rio de Janeiro ${ }^{(1,2)}$
}

\author{
ANDREZZA DA SILVA MACHADO NETO(3); JANIE MENDES JASMIM(4)
}

\begin{abstract}
RESUMO
Este trabalho teve como objetivos caracterizar o sistema produtivo e identificar as principais limitações da produção e comercialização de Heliconia, Alpinia, Etlingera e Anthurium no Estado do Rio de Janeiro. Os dados da pesquisa foram gerados mediante a aplicação de questionário, por meio de visitas e entrevistas pessoais com onze dos quatorze produtores do Estado. A maioria dos produtores está na atividade há cinco anos ou menos (73\%). A atividade é desenvolvida por um pequeno número de produtores, sendo de caráter econômico secundário. O cultivo é feito a pleno-sol (Heliconia, Alpinia e Etlingera) e sob cultivo protegido (Anthurium) predominantemente, com manejo integrado de adubação numa floricultura irrigada, cujas principais limitações são, em ordem decrescente de importância: deficiência na divulgação do produto, no escoamento da produção, na assistência técnica especializada, na estrutura inadequada da Cadeg (Centro de Abastecimento do Estado da Guanabara) e no preço do produto. O mercado de flores tropicais no Rio de Janeiro é um ambiente comercial ainda não consolidado e exigente em ações mercadológicas para o seu desenvolvimento.
\end{abstract}

Palavras-chave: flor tropical, produção, manejo, comércio.

\section{ABSTRACT \\ Profile of production tropical flowers in Rio de Janeiro state}

This work had the objectives of characterizing and identifying the main drawbacks on the production and commercialization of Heliconia, Alpinia, Etlingera e Anthurium in Rio de Janeiro State. The research data were generated by applying questionnaires, through visits and personal interviews with eleven, among the fourteen producers of the State. Most producers $(73 \%)$ are in the activity for a period of five years or less. The activity is developed by a small number of producers, it is of secondary economical character, grown at field conditions (Heliconia, Alpinia and Etlingera) and under a shade house (Anthurium), predominantly, under an integrated fertilization management in a irrigated floriculture, whose main limitations are, in decreasing order of importance: deficiency in advertising the produce, in the produce flow, in specialized technical assistance; CADEG (Centro de Abastecimento do Estado da Guanabara) inadequate structure and the produce price. The market of tropical flowers in Rio de Janeiro appears as a commercial atmosphere not yet consolidated and demanding in marketing actions for its development.

Keywords: tropical flower, production, management, commerce.

\section{INTRODUÇÃO}

As flores tropicais possuem características peculiares que as diferenciam das demais, como exoticidade, coloração contrastante e longevidade pós-colheita. Tais características representam um dos motivos pelos quais têm atraído consumidores, tornando-se uma promessa de rentabilidade satisfatória para diversos produtores. Consequentemente, o cultivo de espécies tropicais é uma parcela do mercado que vem demandando atenção crescente por parte dos especialistas em floricultura, pois vários aspectos da produção e comercialização destas espécies são praticamente desconhecidos, dificultando o exercício da atividade pelos produtores (CASTRO, 2007).

Entre as flores tropicais mais cultivadas no Brasil, o gênero Heliconia L., único da família Heliconiaceae, os gêneros Alpinia e Etlingera (Bastão do imperador), ambos da família Zingiberaceae e o gênero Anthurium (Araceae), merecem destaque (LAMAS, 2004a)

Diversos estados brasileiros vêm desenvolvendo a floricultura tropical, alguns deles como Pernambuco, Ceará e Alagoas se destacam neste setor, seguidos de Rio de Janeiro, Santa Catarina, Bahia e Pará (FRANÇA e MAIA, 2008; BATALHA e BUAINAIN, 2007).

O estado do Rio de Janeiro ocupou, em 2002, a quinta posição em produção de flores no Brasil (AKI E PEROSA, 2002) e é considerado um dos principais fornecedores de flores tropicais para o mercado de São Paulo (VERA, 2008).

A amplitude de climas e solos no Rio de Janeiro possibilita a implantação de cultivos diversificados, tanto de espécies de clima tropical como aquelas de clima temperado, caracterizando-se como vantagem técnica sobre os demais estados brasileiros. Além disso, o Rio de Janeiro conta com um programa de apoio à floricultura no estado, denominado Florescer, mediante o qual, os produtores

\footnotetext{
(1) Recebido para publicação em 27/02/2012 aceito para publicação em 12/04/2012.

(2) Parte da dissertação de mestrado da primeira autora, apresentada ao curso de pós-graduação em Produção Vegetal, Universidade Estadual do Norte Fluminense Darcy Ribeiro/UENF.

(3) Rua Silvio Bastos Tavares, 348, bl 13 apto.103, Pq Rodoviário. Campos dos Goytacazes - RJ CEP:28051-250, andrezzasmachado@yahoo.com.br (4) Avenida Alberto Lamego, 2000 Pq. Califórnia, Campos dos Goytacazes -RJ CEP: 28013-602 - Universidade Estadual do Norte Fluminense Darcy Ribeiro, Centro de Ciências e Tecnologias Agropecuárias - CCTA, Prédio P4 sala 116, janie@uenf.br
} 
interessados em ingressar no setor da floricultura podem contar com um sistema de financiamento de até $100 \%$ do valor orçado para o projeto, assistência técnica através de uma parceria com a Emater-Rio, além de ter acesso a novas tecnologias desenvolvidas e testadas pelas instituições ligadas ao Programa (SEAPPA - RJ, 2004). No entanto, pouco se sabe sobre o sistema de cultivo e as principais limitações da atividade dentro do cenário produtivo e comercial do Estado do Rio de Janeiro.

Diante do potencial produtivo e exportador de flores tropicais do Rio de Janeiro, este trabalho teve como objetivos caracterizar a produção de helicônias, alpínias, bastões do imperador e antúrios no Rio de Janeiro e identificar as principais limitações da produção e comercialização destas espécies.

\section{MATERIAL E MÉTODOS}

A produção de flores tropicais foi diagnosticada por meio de um questionário, aplicado, nas diferentes Regiões produtoras do Estado do Rio de Janeiro, no período de junho de 2007 a setembro de 2009. As visitas foram feitas "in loco" nas quatro Regiões produtoras de flores tropicais, tendo sido identificado um total de quatorze produtores. $\mathrm{O}$ questionário composto por perguntas abertas e fechadas foi dividido em duas partes, A e B, sendo respondido pelos próprios produtores. $\mathrm{Na}$ parte $\mathrm{A}$, composta de perguntas sobre a unidade de produção, foram constatadas as formas de cultivo e manejo de helicônia, alpínia, bastão do imperador e antúrio utilizadas pelos produtores do Estado do Rio de Janeiro. Por meio da parte B, foram identificados e classificados, em ordem decrescente de importância, os fatores que limitam o sistema produtivo e a comercialização das espécies consideradas, conforme a frequência de citação de cada fator apontado pelos produtores entrevistados.

Onze, entre os quatorze produtores de flores tropicais existentes e visitados, foram amostrados, distribuídos nas regiões Médio Paraíba, Centro-Sul, Metropolitana e Noroeste do Estado do Rio de Janeiro, que concordaram em fornecer informações respondendo a questões referentes à condição da propriedade (própria, arrendada); tipo de dependência econômica do produtor em relação à atividade (prioritária, secundária, eventual); área da propriedade; área plantada total; área plantada com flores tropicais; tempo de atuação em floricultura tropical; uso de assistência técnica; tipo de assistência técnica (privada, Emater, prefeituras locais e outros); frequência da assistência técnica; prática de análise de solo; manejo de adubação utilizado; uso e tipo de irrigação; espécies cultivadas; área plantada por espécie (Heliconia sp., Alpinia purpurata, Etlingera elatior e Anthurium andraeanum); forma de propagação das plantas; e espaçamento de plantio.

Cada unidade de produção foi identificada numericamente, de modo que a identidade de cada produtor fosse preservada.

As análises foram realizadas levando-se em consideração as respostas contidas nos questionários, cujos resultados foram comparados por meio de análise descritiva, utilizando-se o MS Excel®, agrupados em tabelas e gráficos, objetivando melhor comparação, discussão e apresentação dos resultados (LOPES et al., 2004).

\section{RESULTADOS E DISCUSSÃO}

\section{Condição dos produtores}

Uma característica bastante peculiar aos produtores de flores e plantas ornamentais do Estado do Rio de Janeiro, observada no Censo da Floricultura Fluminense (EMATER-RIO, 2004), diz respeito ao elevado percentual de produtores na condição de proprietários, não sendo diferente na floricultura tropical. Observou-se que 91\% dos produtores desenvolvem a atividade de floricultura tropical em áreas próprias. Apenas um produtor apresentou a condição de arrendatário, o que corresponde a 9\% do universo amostrado. Tal característica reflete sobre o perfil desta unidade produtiva, dando preferência às espécies cultivadas a pleno sol e de ciclo curto, em virtude do tempo estipulado para o arrendamento.

O cultivo de flores tropicais é desenvolvido economicamente de forma prioritária por apenas $18 \%$ dos produtores. A maioria (73\%) tem o cultivo de flores tropicais como uma atividade secundária na composição da renda familiar e apenas um produtor (9\%) desenvolve a atividade de forma eventual, efetuando operações de manejo e comercialização esporadicamente.

A área ocupada com espécies tropicais representou uma pequena parcela da área total das unidades produtivas (Tabela 1). Segundo os onze produtores amostrados, o cultivo de pequenas áreas é uma medida de precaução contra eventuais prejuízos, em virtude da ausência de informações sobre o mercado de destino destas flores, a velocidade de entouceiramento e a elevada produtividade destas espécies.

\section{Informações sobre a unidade de produção}

Das espécies tropicais estudadas, as helicônias ocupam a maior parcela em área de cultivo, seguidas dos bastões do imperador, antúrios e alpínias (Figura 1). 
Tabela 1. Descrição dos produtores entrevistados, com todos os produtos encontrados em cada unidade produtiva, a área total e a área com espécies tropicais em hectares (ha)

Table 1. Description of the producers interviewed, with all the products found in every productive unit, the total area and the area with tropical species

\begin{tabular}{|c|c|c|c|}
\hline Produtor & $\begin{array}{r}\text { Área } \\
\text { total (ha) }\end{array}$ & $\begin{array}{c}\text { Área com } \\
\text { espécies tropicais } \\
\text { (ha) }\end{array}$ & Descrição dos Produtos \\
\hline 1 & 70 & 25 & $\begin{array}{l}\text { Helicônias, Alpínias, Bastão, Antúrio, Musa vermelha, Gengibre } \\
\text { Ornamental, Tapeinochilo e Folhagens }\end{array}$ \\
\hline 2 & 7,26 & 0,14 & Helicônias, Bastão e Musa vermelha \\
\hline 3 & 290,40 & 0,70 & $\begin{array}{l}\text { Helicônias, Alpínia, Bastão do imperador, Strelítizia e Bovino } \\
\text { de Leite }\end{array}$ \\
\hline 4 & 29,50 & 0,23 & Helicônia e Musa vermelha \\
\hline 5 & 82,28 & 0,20 & $\begin{array}{l}\text { Helicônias;,Alpínia, Musa Vermelha, Gengibre Ornamental e } \\
\text { Eucalipto }\end{array}$ \\
\hline 6 & 14,52 & 2 & Helicônias, Musa Vermelha e Gengibre Ornamental \\
\hline 7 & 264 & 0,65 & $\begin{array}{l}\text { Helicônias, Bastão do imperador, Musa Vermelha, Gengibre } \\
\text { Ornamental, Equinos e Bovino de Leite }\end{array}$ \\
\hline 8 & 8 & 1,5 & Helicônias, Musa Vermelha e Gengibre Ornamental \\
\hline 9 & 15 & 2,1 & Antúrio e Strelítizia \\
\hline 10 & 556,60 & 0,04 & Antúrio, Bovino de Corte e Jacaré \\
\hline 11 & 25 & 3 & $\begin{array}{c}\text { Helicônias, Bastão do Imperador, Gengibre Ornamental, } \\
\text { Hortaliças e Citros }\end{array}$ \\
\hline
\end{tabular}

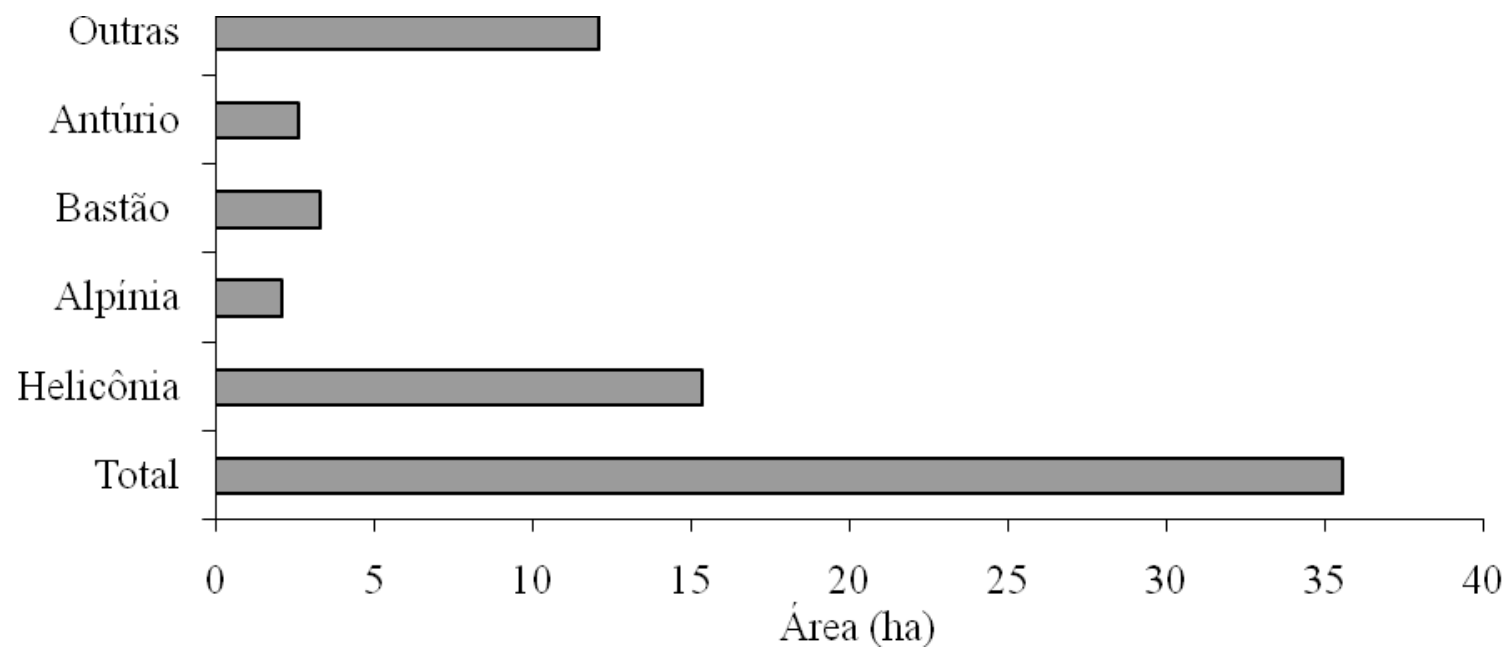

Figura 1. Área cultivada com espécies tropicais no Rio de Janeiro, em hectares.

Figure 1. Area cultivated with tropical species in Rio de Janeiro, in hectares. 
A predominância do cultivo de helicônias sobre as demais espécies tropicais, no Estado do Rio de Janeiro, também é observada em outros estados brasileiros como Pernambuco, Ceará, Alagoas e Minas Gerais (AKI e PEROSA, 2002; JUNQUEIRA e PEETZ, 2002; LANDGRAF e PAIVA, 2009), o que faz com que as helicônias (Heliconia spp.) ocupem a terceira colocação em área cultivada (101,8 ha), no Brasil (JUNQUEIRA e PEETZ, 2002), superadas apenas pelas rosas (426 ha) e crisântemos (234 ha).

O cultivo de bastão do imperador ocupou o segundo lugar em importância no Rio de Janeiro, revelando uma tendência nacional para o incremento da área de produção desta espécie, devido ao seu grande potencial de mercado (LOGES et. al., 2008).

A Figura 2 mostra as áreas de produção de helicônias, alpínias, bastões do imperador e antúrios no Rio de Janeiro, com suas respectivas inflorescências em destaque.

A diferença encontrada entre a área total de cultivo com espécies tropicais e a área cultivada apenas com helicônias, alpínias, bastões e antúrios correspondeu ao cultivo de outras espécies como folhagens, musa e gengibre ornamental (sorvetão).

O exercício da atividade de flores tropicais é recente para $73 \%$ dos produtores que atuam nesta área há cinco, ou menos de cinco anos. Esta grande mobilização pela maioria dos produtores entrevistados, nos últimos cinco anos, está intimamente relacionada à crescente propaganda e incentivos feitos pela Secretaria do Estado do Rio de Janeiro através do programa Florescer, iniciado em 2004 (SEAPPA - RJ, 2004).

Alguns destes produtores migraram de atividades agrícolas tradicionais como cultivo de hortaliças. Outros estão ingressando pela primeira vez na agricultura, o que explica o despreparo com o seu manejo, acarretando grandes prejuízos e uma concorrência desigual com aqueles produtores que atuam há muito tempo na atividade como o produtor número 01 que desenvolve a atividade de floricultura tropical há 45 anos, Figura 3, e que ocupa uma expressiva porção em área cultivada no Estado do Rio de Janeiro (25 ha). Segundo ele, além dos incentivos que o Estado vem oferecendo à atividade, ele deveria mostrar os pontos de estrangulamento do setor, como a presença de um mercado com alta demanda, porém de difícil acesso e que, para serem competitivos, os produtores precisariam investir em volume e qualidade da produção.

As demais áreas de cultivo de flores tropicais do Estado, quando somadas, correspondem a 10,56 hectares, tornando praticamente impossível usar o volume de produção como veículo de competitividade.

Todavia, o trabalho associativista, a busca por outros mercados e o investimento na qualidade do produto têm sido algumas das alternativas encontradas pelos produtores da Região Médio Paraíba para viabilizar a produção de flores tropicais.

\section{Características relacionadas ao manejo}

Todos os produtores de flores tropicais entrevistados receberam assistência técnica durante o período de implantação dos cultivos, mas apenas quatro propriedades possuem continuidade deste serviço ao longo do ciclo produtivo. Tal característica diferenciou a floricultura tropical das demais atividades do setor da floricultura e plantas ornamentais, pois, segundo o Censo da Floricultura e Plantas Ornamentais do Rio de Janeiro, mais da metade dos produtores não recebeu qualquer tipo de assistência técnica $(61,08 \%)$ em seus respectivos sistemas de cultivo (EMATER- RIO, 2004).

Provavelmente, o pequeno número de produtores e a intensiva busca por informações e técnicas de cultivo sejam os fatores que explicam a predominância de assistência técnica nas unidades produtoras de flores tropicais. Entre as fontes de fornecimento de assistência técnica, temos a prefeitura local (64\%), Emater-Rio (9\%), privada (18\%) e outras $(9 \%)$ como universidades e órgãos de pesquisa que assessoram os produtores de flores tropicais no Rio de Janeiro.

Antes da implantação dos cultivos, nove dos onze produtores entrevistados fizeram a análise do solo, restando apenas dois que não o fizeram, correspondendo a $82 \%$ e $18 \%$, respectivamente, da população amostrada.

Todavia, embora a maioria tenha feito a análise de solo antes do plantio, 1/3 destes produtores não a usaram como base para a recomendação de adubação, provavelmente pela descontinuidade do fornecimento dos serviços de assistência técnica.

Uma característica relevante, encontrada nos sistemas produtivos de flores tropicais, no estado do Rio, foi a preocupação com o meio ambiente e a busca por uma agricultura sustentável. Entre os onze produtores entrevistados, oito deles utilizam em suas propriedades um sistema integrado de manejo mediante o uso de um mix de insumos, químicos e orgânicos, apenas um utilizou o sistema químico e os dois restantes utilizam o sistema de manejo orgânico.

O objetivo de todos os produtores que utilizam o sistema de manejo integrado é alcançar a máxima substituição de elementos químicos por elementos orgânicos aproveitando os resíduos da própria propriedade, como restos vegetais, por exemplo, que, ao invés de serem simplesmente descartados, são reincorporados ao sistema produtivo como um elemento complementar à nutrição das plantas e ao equilíbrio da temperatura, umidade e microbiota do solo.

Com relação à forma de fornecimento hídrico, apenas um produtor, entre todos os entrevistados, não irriga sua área de cultivo, correspondendo a $9 \%$ do total amostrado. Tal comportamento se deve à localização desta unidade produtiva, situada numa região úmida com médias anuais de $60-70 \%$ de umidade relativa.

O sistema de irrigação predominante, em $91 \%$ das áreas de cultivo, é do tipo aspersão alta e os $9 \%$ restantes irrigam manualmente. Tal característica é um fator preocupante no que diz respeito à qualidade fitossanitária das plantas, pois a irrigação por aspersão alta favorece o acúmulo de umidade nas folhas e inflorescências, criando um ambiente propício ao ataque de doenças, prejudicando a produção tanto em termos quantitativos quanto qualitativos (ASSIS et. al., 2002). Além disso, inicialmente, quando as plantas estão jovens e de menor porte, o sistema funciona muito bem no que se refere ao raio de cobertura da irrigação, mas, à medida que as plantas crescem e formam touceiras, 

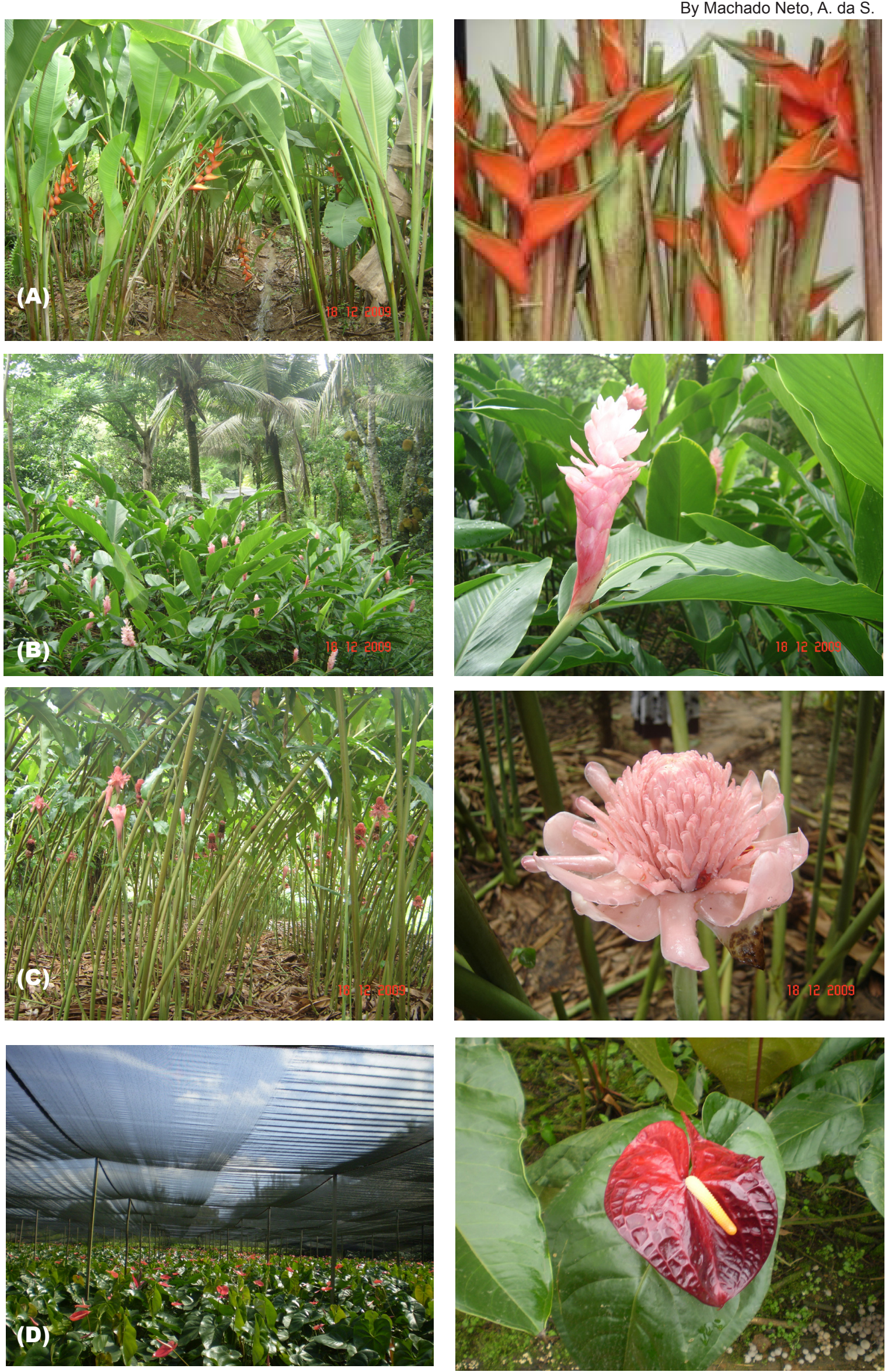

Figura 2. Unidades de produção: (A) Heliconia bihai; (B) Alpinia purpurata 'Pink Ginger'; (C) Etlingera elatior 'Porcelain' e (D) Anthurium andraeanum 'Iguape'.

Figure 2. Production units: (A) Heliconia bihai; (B) Alpinia purpurata 'Pink Ginger'; (C) Etlingera elatior 'Porcelain'e (D) Anthurium andraeanum 'Iguape'. 
esta eficiência diminui, reduzindo assim a oferta hídrica necessária para seu desenvolvimento. O ideal é que este tipo de irrigação seja evitado e substituído por um sistema de irrigação por gotejamento (LAMAS, 2004b).

\section{Principais espécies}

As principais espécies cultivadas no Rio de Janeiro, dentro dos gêneros Heliconia, Alpinia, Etlingera e Anthurium, estudados neste trabalho, são: Heliconia bihai, Heliconia psittacorum, Heliconia rostrata, Heliconia latispatha, Heliconia wagneriana, Alpinia purpurata ('Red
Ginger' e 'Pink Ginger'), Etlingera elatior ('Red Torch', Pink Torch' e 'Porcelain') e Anthurium andraeanum.

A importância de tais espécies se deveu ao fato de estarem presentes na maioria das unidades produtoras de flores tropicais do Rio de Janeiro. Entre os onze produtores amostrados, nove produzem Heliconia bihai, o que implicou um elevado volume desta espécie a ser ofertada no mercado de flores do Rio de Janeiro. Observou-se que há um elevado número de produtores dentro da população amostrada que cultiva as mesmas espécies, como, por exemplo, Heliconia bihai, Heliconia psittacorum e Heliconia rostrata (Figura 4).

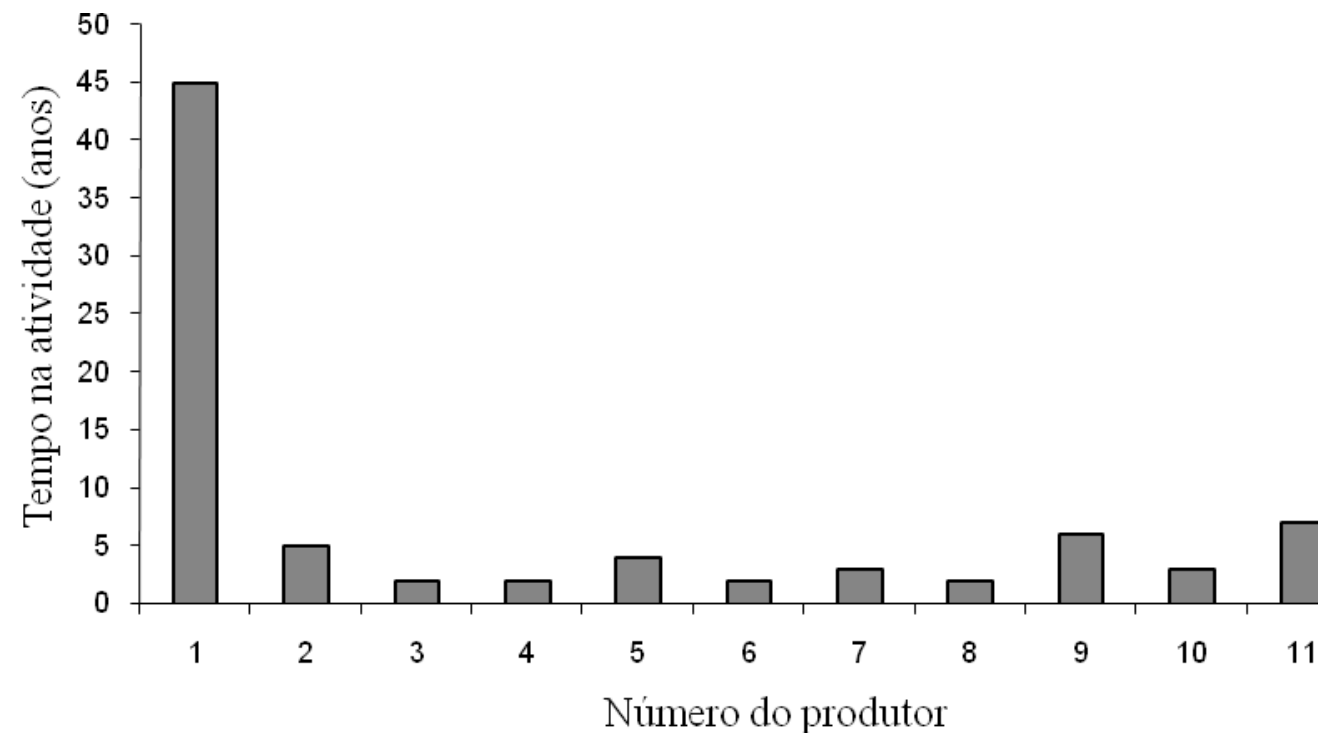

Figura 3. Tempo na atividade de floricultura tropical de cada produtor no estado do Rio de Janeiro. Figure 3. Time in the activity of tropical flowers of every producer in the state of Rio de Janeiro.

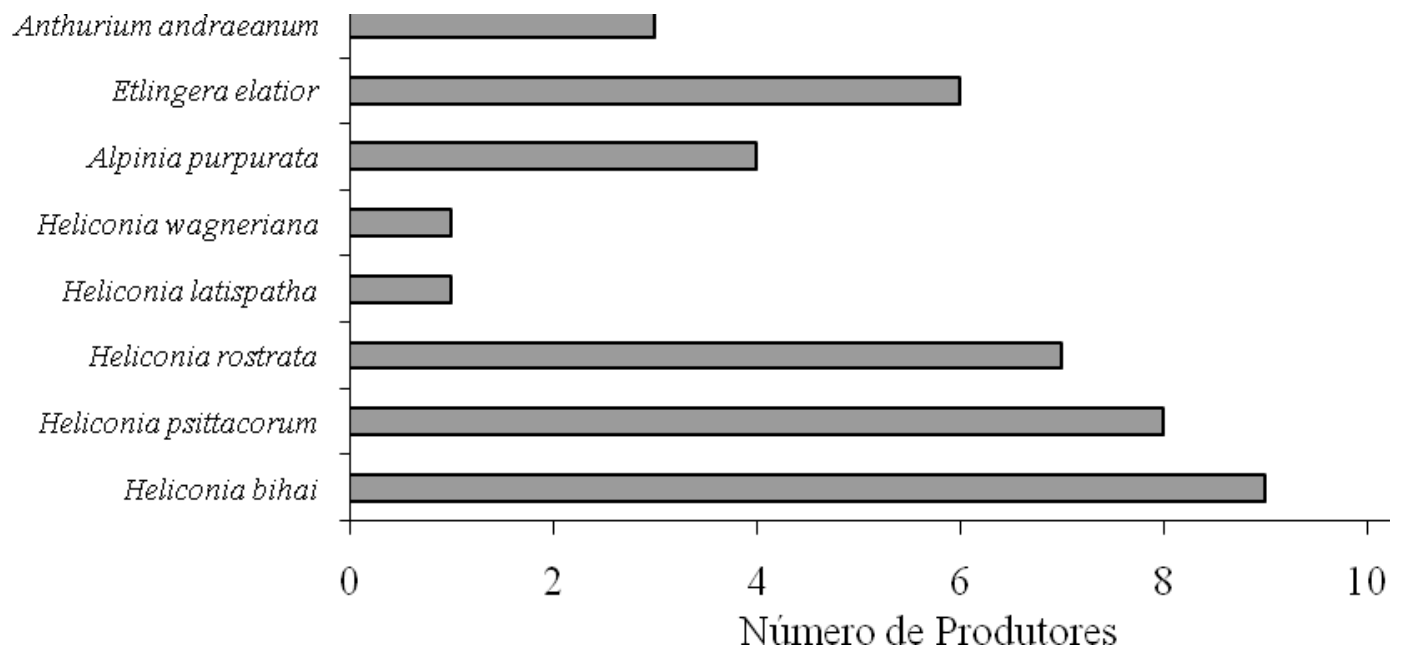

Figura 4. Principais espécies encontradas nas unidades de cultivo de flores tropicais no estado do Rio de Janeiro.

Figure 4. Main species found in the units of tropical flowers growing in the state of Rio de Janeiro.

Em termos de mercado, o surgimento de produtores com as mesmas espécies poderia estabelecer um ambiente de competitividade por preço e qualidade, o que beneficiaria os consumidores e tornaria o Estado ainda mais competitivo no cenário nacional. No entanto, não é isso o que vem acontecendo, pois as áreas de cultivo dos demais produtores são bem menores que a do produtor número 01 para estas mesmas espécies. Dos 5,54 hectares obtidos pelo somatório das áreas de cultivo com Heliconia bihai, Heliconia psittacorum e Heliconia rostrata no Rio de Janeiro, Tabela 2, 3,5 hectares pertencem ao produtor número 01. Trata-se de um produtor pioneiro dentro da 
atividade, que, além de possuir uma expressiva porção em área cultivada, ocupa uma significativa porção do mercado interno do Rio de Janeiro.

$\mathrm{O}$ investimento em outros materiais vegetais, espécies e variedades, poderia ser uma alternativa de rentabilidade para os produtores, trazendo diversificação à produção de flores tropicais do Rio de Janeiro e mais opções de produtos aos consumidores.

\section{Forma de propagação}

Todos os produtores compraram e utilizaram rizomas na propagação de helicônias, alpínias e bastões do imperador, como o recomendado por diversos autores na literatura (LAMAS, 2004a; LAMAS, 2004b; CRILEY, 1995; CASTRO, 1995), e mudas provenientes de cultivo in vitro na implantação do cultivo de antúrio.

Após a implantação dos cultivos, o método de divisão de touceiras é a forma predominante de obtenção de mudas em todas as propriedades, quando se deseja, por exemplo, a ampliação da área de produção.

\section{Espaçamento}

Os dados da Tabela 3 mostram os espaçamentos observados nas áreas de cultivo no Rio de Janeiro. Todas as espécies foram plantadas no sistema de fileira simples, porém o plantio de antúrios foi feito em canteiros de 1,20 -1,40 m de largura, cujo comprimento variou de acordo com a área disponível para o plantio, respeitando o limite máximo de $50 \mathrm{~m}$, sugerido por TOMBOLATO et al. (2002).

$\mathrm{O}$ dimensionamento variou em algumas propriedades para a mesma espécie avaliada. No entanto, nos plantios de helicônias, foram observadas as maiores variações, se comparado com o sugerido pela literatura (CASTRO, 1995).

\section{Fatores limitantes}

As principais limitações apontadas pelos produtores no setor de flores tropicais no Rio de Janeiro foram, em ordem de importância: deficiência na divulgação do produto, no escoamento da produção, na assistência técnica especializada, na estrutura da Cadeg (Centro de Abastecimento do Estado da Guanabara) e no baixo valor recebido pelo produto.

Todos os produtores acreditam no potencial de mercado do setor de flores tropicais, argumentando que um trabalho efetivo de divulgação poderia mostrar aos consumidores a tendência nacional do uso de espécies exóticas, com cores atraentes e uma longa vida pós-colheita, na confecção de arranjos, buquês, decorações de eventos e interiores em geral. Tal estratégia, provavelmente, promoveria uma mudança no comportamento do consumidor, que, na maioria dos casos, desconhece as flores tropicais, estabelecendo uma correlação positiva com o fator escoamento da produção pelo incremento nas vendas à medida que novos consumidores forem apresentados aos produtos.

Em algumas unidades produtivas, as perdas, de até $80 \%$ para algumas espécies, são consequência da falta de transporte para o escoamento da produção e, em outros casos, porque o plantio foi efetuado sem conhecimento prévio do mercado consumidor. Situações como estas devem e podem ser previstas no período de implantação ou de expansão dos cultivos quando uma pesquisa de mercado anteceder o planejamento da produção, e uma articulação adequada entre a produção e canais de escoamento minimizaria o prejuízo ao longo do período de produtivo.

A carência de assistência técnica especializada foi o terceiro fator mais apontado pelos produtores, considerado fator limitante ao sistema produtivo. Os agricultores lamentaram as recomendações técnicas equivocadas e os prejuízos delas decorrentes, o que parece ser uma deficiência de todo o estado (EMATER-RIO, 2004).

Há pouca informação sobre o segmento de flores tropicais (ANEFALOS et. al., 2009). Tal histórico explica a ausência de profissionais especializados em floricultura, não apenas no Rio de Janeiro, como em todo território nacional, visto que, segundo AKI e PEROSA (2002), se trata de uma atividade recente.

Um dos maiores canais de escoamento da produção de flores tropicais do Rio de Janeiro é a Cadeg, onde as flores são comercializadas pelos produtores, tanto no atacado, quanto no varejo. Os produtores apontaram os custos para a exposição de seus produtos na Central de abastecimento (Cadeg) como fator limitante, pois, além dos gastos com a aquisição ou aluguel do box e condomínio, os expositores precisam pagar estacionamento. Além disso, a estrutura é relativamente precária, as compras são feitas exclusivamente pela visualização dos produtos, o comércio eletrônico é quase inexistente, não há definição de critérios de qualidade dos produtos e a padronização é bastante limitada (CARVALHO e CHIANCA, 2002).

O baixo valor recebido pelo produto é um fator prejudicial à rentabilidade do setor. Em alguns casos, a produção de inflorescências orgânicas, a venda na forma de arranjos e a classificação adotando padrões de qualidade têm sido

Tabela 2. Área em hectare (ha), cultivada com as principais espécies tropicais do Rio de Janeiro Table 2. Area in hectare (ha), cultivated with the main species tropical of Rio de Janeiro

\begin{tabular}{cc}
\hline Espécie & Área (ha) \\
\hline Heliconia psittacorum & 2,81 \\
Heliconia bihai & 1,93 \\
Heliconia rostrata & 0,80 \\
Heliconia latispatha & 1,50 \\
Heliconia wagneriana & 0,32 \\
Alpinia purpurata & 2,10 \\
Etlingera elatior & 3,32 \\
Anthurium andraeanum & 2,64 \\
\hline
\end{tabular}


Tabela 3. Espaçamentos utilizados nas unidades de cultivo de flores tropicais no Rio de Janeiro Table 3. Spacings units used in the cultivation of tropical flowers in Rio de Janeiro

\begin{tabular}{|c|c|c|c|c|c|c|c|c|}
\hline \multirow{2}{*}{$\begin{array}{l}\text { Unidades } \\
\text { Produtivas }\end{array}$} & \multicolumn{2}{|c|}{ Heliconia bihai } & \multicolumn{2}{|c|}{ Heliconia psittacorum } & \multicolumn{2}{|c|}{ Heliconia rostrata } & \multicolumn{2}{|c|}{ Heliconia latispatha } \\
\hline & Espaçamento & $\begin{array}{l}\text { Plantas/ } \\
\text { Hectare }\end{array}$ & Espaçamento & $\begin{array}{l}\text { Plantas/ } \\
\text { Hectare }\end{array}$ & Espaçamento & $\begin{array}{l}\text { Plantas/ } \\
\text { Hectare }\end{array}$ & Espaçamento & Plantas/Hectare \\
\hline 1 & $2,0 \times 2,5$ & 2000 & $1,5 \times 1,5$ & 4444 & $2,0 \times 2,5$ & 2000 & $2,0 \times 2,5$ & 2000 \\
\hline 2 & $2,0 \times 2,0$ & 2500 & $0,5 \times 0,8$ & 25000 & --- & --- & --- & --- \\
\hline 3 & $2,0 \times 2,0$ & 2500 & --- & --- & $2,5 \times 2,0$ & 2000 & --- & --- \\
\hline 4 & $1,5 \times 1,5$ & 4444 & $1,5 \times 1,5$ & 4444 & --- & --- & --- & --- \\
\hline 5 & $2,0 \times 1,5$ & 3333 & $2,0 \times 1,5$ & 3333 & $2,0 \times 1,5$ & 3333 & --- & --- \\
\hline 6 & $2,5 \times 2,5$ & 1600 & $2,5 \times 2,5$ & 1600 & --- & --- & --- & --- \\
\hline 7 & $2,0 \times 2,0$ & 2500 & $1,5 \times 1,5$ & 4444 & $1,5 \times 2,0$ & 3333 & --- & --- \\
\hline 8 & $2,0 \times 2,0$ & 2500 & $1,5 \times 1,0$ & 6666 & $2,0 \times 2,0$ & 2500 & --- & --- \\
\hline 9 & --- & --- & --- & --- & --- & --- & --- & --- \\
\hline 10 & --- & --- & --- & --- & --- & --- & --- & --- \\
\hline 11 & $1,0 \times 3,0$ & 3333 & $1,0 \times 1,5$ & 6666 & $1,0 \times 3,0$ & 3333 & --- & --- \\
\hline
\end{tabular}

\begin{tabular}{|c|c|c|c|c|c|c|c|c|}
\hline \multirow{2}{*}{$\begin{array}{c}\text { Unidades } \\
\text { Produtivas }\end{array}$} & \multicolumn{2}{|c|}{ Heliconia wagneriana } & \multicolumn{2}{|c|}{ Alpinia purpurata } & \multicolumn{2}{|c|}{ Etlingera elatior } & \multicolumn{2}{|c|}{ Anthurium andraeanum } \\
\hline & Espaçamento & $\begin{array}{l}\text { Plantas/ } \\
\text { Hectare }\end{array}$ & Espaçamento & $\begin{array}{l}\text { Plantas/ } \\
\text { Hectare }\end{array}$ & Espaçamento & $\begin{array}{l}\text { Plantas/ } \\
\text { Hectare }\end{array}$ & Espaçamento & $\begin{array}{l}\text { Plantas/ } \\
\text { Hectare }\end{array}$ \\
\hline 1 & --- & --- & $2,0 \times 2,5$ & 2000 & $3,0 \times 3,0$ & 1111 & $0,4 \times 0,4$ & 46375 \\
\hline 2 & $2,0 \times 2,0$ & 2500 & $3,0 \times 2,0$ & 1666 & $3,0 \times 3,0$ & 1111 & --- & --- \\
\hline 3 & --- & --- & --- & --- & $3,0 \times 3,0$ & 1111 & --- & --- \\
\hline 4 & --- & --- & --- & --- & --- & --- & --- & --- \\
\hline 5 & --- & --- & $2,0 \times 2,0$ & 2500 & $2,0 \times 3,0$ & 1666 & --- & --- \\
\hline 6 & --- & --- & --- & --- & --- & --- & --- & --- \\
\hline 7 & --- & --- & --- & --- & $2,0 \times 2,0$ & 2500 & --- & --- \\
\hline 8 & --- & --- & --- & --- & --- & --- & --- & --- \\
\hline 9 & --- & --- & --- & --- & --- & --- & $0,3 \times 0,3$ & 82500 \\
\hline 10 & --- & --- & --- & --- & --- & --- & $0,5 \times 0,5$ & 29750 \\
\hline 11 & --- & --- & --- & --- & $1,0 \times 3,0$ & 3333 & --- & --- \\
\hline
\end{tabular}

medidas estratégicas adotadas por alguns produtores para agregar valor ao produto e aumentar a rentabilidade por unidade de área.

\section{CONCLUSÃO}

A produção comercial de helicônia, alpínia, bastão do imperador e antúrio é uma atividade recente (cinco anos ou menos) para a maioria dos produtores do estado do Rio de Janeiro, cujas principais limitações, conforme a frequência de citação pelos produtores, são, em ordem decrescente de importância: deficiência na divulgação do produto, no escoamento da produção, na assistência técnica especializada, estrutura inadequada da Cadeg (Centro de Abastecimento do Estado da Guanabara) e o preço do produto.

Todavia, diante do potencial produtivo das espécies tropicais, faz-se necessário o desenvolvimento de uma estruturação do mercado, seguida de um planejamento estratégico de expansão, pela adoção de medidas efetivas que prezem pela capacitação dos agricultores e técnicos e pela promoção desses produtos no mercado.

\section{REFERÊNCIAS}

AKI, A. \& PEROSA, J. M. Aspectos da produção e consumo de flores e plantas ornamentais no Brasil. Revista
Brasileira de Horticultura Ornamental, Campinas- SP, v. 8, p.13-23. 2002.

ANEFALOS, C. L.; TOMBOLATO, A. F. C.; RICORDI, A. Cadeia produtiva de flores tropicais: aspectos gerais da produção e comercialização. In: XVII CONGRESSO BRASILEIRO DE FLORICULTURA E PLANTAS ORNAMENTAIS IV CONGRESSO BRASILEIRO DE CULTURA DE TECIDOS DE PLANTAS, Resumos... Aracajú-SE, 2009. CD-ROM.

ASSIS, S. M. P., MARIANO, R. R. L., GODIM JÚNIOR, M. G. C., MENEZES, M., ROSA, R. C. T. da. (2002) Doenças e pragas das helicônias. Universidade Federal Rural de Pernambuco, Recife-PE, 102 p.

BATAlHA, M. O. \& BUAINAIN, A. M. Cadeias produtivas de flores e mel. Série Agronegócios. v.9, p.4977. 2007.

CARVALHO, R. L. de \& CHIANCA, G. K. A produção de flores e plantas ornamentais do Estado do Rio de Janeiro: evolução recente, desafios e perspectivas. Pesquisa Agropecuária e Desenvolvimento Sustentável. v.1, p.97112. 2002.

CASTRO, A. C. R. de. Deficiência de macronutrientes 
em Heliconia psittacorum 'Golden Torch'. Recife-PE, Universidade Federal Rural de Pernambuco, 2007. 102 p. Dissertação Mestrado.

CASTRO, C.E.F. de. Heliconia para exportação: aspectos técnicos da produção. Brasília: MAARA-SDRFRUPEX/ ISPI, (FRUPEX. Publicações Técnicas, 16). Brasília: Embrapa-SPI, 43p, 1995.

CRILEY, R.A. Propagation of Zingiberaceae and Heliconiaceae. Revista Brasileira de Horticultura Ornamental. Campinas, v.1, p.14-21, 1995.

EMATER - RIO. Censo da floricultura no Estado do Rio de Janeiro. 2004. CD-ROM.

FRANÇA, C. A. M. de e MAIA, M. B. R. Panorama do agronegócio de flores e plantas ornamentais no Brasil. In: XLVI CONGRESSO DA SOCIEDADE BRASILEIRA DE ECONOMIA, ADMINISTRAÇÃO E SOCIOLOGIA RURAL, 2008. Anais... Rio Branco-Acre, CD-ROM.

JUNQUEIRA, A. H. \& PEETZ, M. S. Os polos de produção de flores e plantas ornamentais do Brasil: uma análise do potencial exportado. Revista Brasileira de Horticultura Ornamental. Campinas-SP, v.8, n. 1/2 , p. 25-47. 2002.

LAMAS, A. M. Flores: produção, pós-colheita e mercado. Fortaleza: Instituto Frutal, 2004a. 109p.

LAMAS, A. M. Curso: Flores Tropicais - tecnologia de produção. Tabatinga-AM, 2004b, 65p.
LANDGRAF, P.R.C. e PAIVA, P.D. de O. Produção de flores cortadas no estado de Minas Gerais. Revista Ciência e Agrotecnologia, vol.33, n.1, p.120-126, jan./fev., 2009.

LOGES, V.; COSTA, A. S.; GUIMARÃES, W. N. R.; TEIXEIRA, M. C. F. Potencial de mercado de Bastão-doImperador e Sorvetão. Revista Brasileira de Horticultura Ornamental, Campinas-SP, v.14, n. 1, p.15-22, 2008.

LOPES M.A., LIMA A.L.R., CARVALHO F. de M., REIS R.P., SANTOS I.C., SARAIVA F.H. Controle gerencial e estudo da rentabilidade de sistemas de produção de leite na região de Lavras (MG) Revista Ciência e Agrotecnologia, vol.28, p.883-892, 2004.

Secretaria de Agricultura, Pecuária, Pesca e Abastecimento do Estado do Rio de Janeiro (SEAPPA - RJ). Programa de Apoio à Floricultura no Estado do Rio de Janeiro - FLORESCER. 2004. Disponível em: <http://www. agricultura.rj.gov/florescer $>$ Acesso em: 13 março 2009.

TOMBOLATO, A. F. C.; RIVAS, E. B.; COUTINHO, L. N.; BERMANN, E. C.; IMENES, S. D. L.; FURLANI, P. R.; CASTRO, C. E. F.; MATTHES, L. A. F.; SAES, L. A.; COSTA, A. M. M.; DIAS-TAGLIACOZZO, G. M.; LEME, J. M. O cultivo de antúrio: produção comercial. Boletim Técnico. Instituto Agronômico de Campinas, CampinasSP, v. 194, p.1-47, 2002.

VERA, R. M. Diagnóstico do mercado de flores tropicais. Revista Brasileira de Horticultura Ornamental, Campinas-SP, v. 14, p. 35-36, 2008. 